\title{
Orders from Uninorms on Bounded Lattices: Some Perspectives
}

\author{
Vikash Kumar Gupta ${ }^{a}$ and ${ }^{*}$ Balasubramaniam Jayaram $^{a}$ \\ ${ }^{a}$ Department of Mathematics, Indian Institute of Technology Hyderabad, INDIA. \\ ma17resch01002@iith.ac.in, jbala@math.iith.ac.in
}

\begin{abstract}
Recently many works have proposed different ways of obtaining orders from associative fuzzy logic operations. While the order $\sqsubseteq$ investigated by Karaçal and Kesicioğlu [10] was modified in [6] to obtain orders on uninorms, this order relation $\preceq$ was based on the sub-domains of the arguments. Recently in [9], a property called Quasi-Projectivity (QP) was shown to be important to obtain an order from the relation $\sqsubseteq$ investigated in [10] and showed that all t-norms, $\mathrm{t}$-conorms and nullnorms satisfy (QP) giving rise to posets, when the underlying domain is $[0,1]$, while not all classes of uninorms satisfied (QP). Many constructions of uninorms $U$ exist on bounded lattices, which unlike $[0,1]$, may neither be total nor complete. In this work, we investigate the satisfaction of (QP) for these constructions. Our study shows that interestingly, almost all existing constructions satisfy (QP) and hence give rise to posets. Further, the orders obtained based on $\sqsubseteq$ and $\preceq$ differ majorly, with the $\sqsubseteq$ relation consistently giving rise to richer order-theoretic structures. This study further merits attention since it offers an alternate perspective that a uninorm $U$ on a lattice $(\mathbb{L}, \leq)$ can be made to be seen as a t-norm on the $U$-poset obtained $(\mathbb{L}, \sqsubseteq U)$.
\end{abstract}

Keywords: Uninorms, T-norms, Bounded Lattice, Posets, Quasi-Projectivity.

\section{Introduction}

In recent years, many works have appeared that propose order from basic fuzzy logic connectives, see for instance, [10],[6], [1] ,[12],[8], [7] . Given a bounded lattice $(\mathbb{L}, \leq, 0,1)$, Karaçal and Kesicioğlu [10] were the first ones to investigate whether the following relation, proposed by Clifford [5], would give rise to an order on $\mathbb{L}$ based on a given $\mathrm{t}$-norm $T$ on $\mathbb{L}$ :

$$
x \sqsubseteq y \Longleftrightarrow T(x, \ell)=y, \text { for some } \ell \in \mathbb{L} .
$$

While $\sqsubseteq$ gave rise to orders based on both t-norms and $\mathrm{t}$-conorms, to obtain order from a uninorm this relation was further generalised by Ertuğrul et al. [6].

Definition 1 ([11]). Let $(\mathbb{L}, \leq, 0,1)$ be a bounded lattice. A commutative, associative, non-decreasing in each variable function $U: \mathbb{L}^{2} \rightarrow \mathbb{L}$ is called a uninorm if there is an identity element $e \in \mathbb{L} \backslash\{0,1\}$, i.e., $U(e, x)=x$ for all $x \in \mathbb{L}$.

Definition 2 ([6]). Let $(\mathbb{L}, \leq, 0,1)$ be a bounded lattice and $U$ be a uninorm on $\mathbb{L}$ with an identity element $e \in \mathbb{L} \backslash\{0,1\}$. Define the following relation: For every $x, y \in \mathbb{L}$

$$
x \preceq_{U} y \Leftrightarrow \begin{cases}\text { if } \quad & x, y \in[0, e] \text { and there exists } k \in[0, e] \\ & \text { such that } U(k, y)=x \text { or }, \\ \text { if } \quad & x, y \in[e, 1] \text { and there exists } \ell \in[e, 1] \\ & \text { such that } U(x, \ell)=y \text { or }, \\ \text { if } \quad(x, y) \in \mathbb{L}^{2} \backslash\left\{[0, e]^{2} \cup[e, 1]^{2}\right\} \\ \\ \text { and } x \leq y .\end{cases}
$$

The relation $\preceq_{U}$ is a partial order on $\mathbb{L}$ for any uninorm $U$.

\subsection{Motivation for of this work}

While $\preceq_{U}$ is both a generalisation of $\sqsubseteq$ and gives a partial order on $\mathbb{L}$ for any uninorm $U$, it is not bereft of some disadvantages, as listed below:

(i) The definition of order is dependent on the way the uninorm $U$ is defined on its subdomains. 
(ii) To relate a pair of elements $x, y$, there are further restrictions on the domain from which the second argument of the $U$ can come from.

(iii) The following Proposition 1 shows that we cannot obtain any richer order-theoretic structure from $\preceq_{U}$ than the bounded lattice $\mathbb{L}$ that we begin with. For instance, if $(\mathbb{L}, \leq)$ is not a chain then the partial ordered set $\left(\mathbb{L}, \preceq_{U}\right)$ is also not a chain.

Proposition 1 ([6], Propositions 3). Let $(\mathbb{L}, \leq, 0,1)$ be a bounded lattice and $U$ be a uninorm on $\mathbb{L}$ with an identity element $e \in \mathbb{L} \backslash\{0,1\}$. If $x \preceq_{U}$ y for any $x, y \in$ $\mathbb{L}$, then $x \leq y$.

Recently in [9], a property called Quasi-Projectivity (QP) of an associative operation was shown to be important to obtain an order from the relation $\sqsubseteq$ investigated in [10].

Definition 3 ([9]). Let $\mathbb{P} \neq \emptyset$. An $F: \mathbb{P} \times \mathbb{P} \rightarrow \mathbb{P}$ is said to satisfy the Quasi-Projection property, iffor any $x, y, z \in \mathbb{P}$,

$$
F(x, F(y, z))=z \Longrightarrow F(y, z)=z .
$$

Theorem 1 (Cf. [9], Theorem 2.4). Let $\mathbb{P} \neq \emptyset$ and $F: \mathbb{P} \times \mathbb{P} \rightarrow \mathbb{P}$ be an associative operation with a left identity. Let the relation $\sqsubseteq_{F}$ on $\mathbb{P}$ be as defined in (1), with $F$ instead of $a T$. Then the following are equivalent:

(i) $\left(\mathbb{P}, \sqsubseteq_{F}\right)$ is a poset.

(ii) F satisfies $(\mathbf{Q P})$.

It is clear then that, since a t-norm, t-conorm or a uninorm is both associative and has a neutral element, it is sufficient to check for the satisfaction of $(\mathbf{Q P})$ on its domain.

While this result helps us in employing the relation $\sqsubseteq$ and thus getting over the first two drawbacks discussed above, however, it was shown that not all classes of uninorms over $[0,1]$ satisfy $(\mathbf{Q P})$.

Many constructions of uninorms $U$ exist on bounded lattices, which unlike $[0,1]$, may neither be total nor complete. In this work, we intend to investigate the satisfaction of $(\mathbf{Q P})$ for these constructions.

\subsection{Contributions of this work}

Our study makes the following contributions:

(i) Note that almost all constructions of uninorms $U$ proposed on bounded lattices are done regionwise. By presenting fairly general and unifying results discussing the satisfaction of $(\mathbf{Q P})$ on these different regions, we show that almost all the existing constructions satisfy $(\mathbf{Q P})$.

(ii) We show that the orders obtained based on $\sqsubseteq$ and $\preceq$ differ majorly, with the $\sqsubseteq$ relation consistently giving rise to richer order-theoretic structures.

(iii) An interesting fall out of this study is the rise of an alternate perspective. Recently there have been a spate of works on constructing t-norms on bounded posets/lattices. Our study shows that a uninorm $U$ on the original lattice $(L, \leq)$ can be made to be seen as a t-norm on the obtained poset $\left(L, \sqsubseteq_{U}\right)$.

\section{Uninorms and (QP): Some General Results}

We begin this section with some general results which are useful in proving the results given in Section 2.1.

Remark 1. Let $(\mathbb{L}, \leq, 0,1)$ be a bounded lattice.

(i) A pair of elements $a, b \in \mathbb{L}$ is said to be incomparable, denoted $a \| b$, if neither $a \leq b$ nor $b \leq a$.

(ii) If $a, b \in \mathbb{L}$ such that $a \leq b$ then by the interval $[a, b]$ we denote the following set:

$$
[a, b]=\{x \in \mathbb{L} \mid a \leq x \leq b\} .
$$

(iii) Let $e \in \mathbb{L} \backslash\{0,1\}$. We use the following notations in this work: $A(e)=[0, e] \times[e, 1] \cup[e, 1] \times[0, e]$ and $I_{e}=\{x \in \mathbb{L} \mid e \| x\}$.

(iv) Note that, on a bounded lattice $\mathbb{L}$ with an $e \in \mathbb{L} \backslash$ $\{0,1\}$, we can partition $\mathbb{L}$ into the following nonoverlapping regions, viz., $\mathbb{L}=[0, e) \cup\{e\} \cup I_{e} \cup$ $(e, 1]$.

Proposition 2. Let $(\mathbb{P}, \leq, 0,1)$ be a bounded poset and $F: \mathbb{P} \times \mathbb{P} \rightarrow \mathbb{P}$.

(i) Let one of the following be true:

$$
\begin{aligned}
& F(\alpha, \beta) \geq \beta, \text { for all } \alpha, \beta \in \mathbb{P}, \text { or } \\
& F(\alpha, \beta) \leq \beta, \text { for all } \alpha, \beta \in \mathbb{P} .
\end{aligned}
$$

Then $F$ satisfies $(\mathbf{Q P})$ over $\mathbb{P}^{2}$.

(ii) Let $F$ be increasing in the first variable w.r.t. $\leq$ and let one of the following be true:

$$
\begin{aligned}
& F(0, \beta)=\beta \text { for all } \beta \in \mathbb{P}, \text { or } \\
& F(1, \beta)=\beta \text { for all } \beta \in \mathbb{P} .
\end{aligned}
$$

Then $F$ satisfies $(\mathbf{Q P})$ over $\mathbb{P}^{2}$. 


\subsection{Uninorms and satisfaction of $(\mathrm{QP})$}

Based on the partition given in Remark 1 (iv), any function $F: \mathbb{L} \times \mathbb{L} \rightarrow \mathbb{L}$ can be seen as being defined on the corresponding subdomains of Cartesian products obtained from the pieces of this partition. Especially, in the construction of uninorms $U$ on $\mathbb{L}$ the following domain divisions of $\mathbb{L} \times \mathbb{L}$ are typically employed: $[0, e]^{2}, A(e), I_{e} \times \mathbb{L}, \mathbb{L} \times I_{e},[e, 1]^{2}$ (see Fig. 1).

Thus we investigate the satisfaction of $(\mathbf{Q P})$ in each of the 9 subdomains of $\mathbb{L} \times \mathbb{L}$. Towards this end, we restrict the value $U$ takes on these different subdomains, say $X \times Y$, and show that $U$ satisfies $(\mathbf{Q P})$ on $X \times Y$, i.e., we show that $U$ satisfies $(\mathbf{Q P})$ for every triple $(x, y, z)$ when $(x, y) \in X \times Y$ and $z \in \mathbb{L}$.

Theorem 2. Let $(\mathbb{L}, \leq, 0,1)$ be a bounded lattice and $U$ be a uninorm on $\mathbb{L}$ with an identity element $e \in$ $\mathbb{L} \backslash\{0,1\}$. Then the following are true:

(i) For every triple $(x, y, z) \in \mathbb{L}^{3}$ such that $e \in\{x, y\}$, $U$ satisfies $(\mathbf{Q P})$.

(ii) $U$ satisfies $(\mathbf{Q P})$ on the subdomain $[0, e]^{2}$, i.e., if $(x, y, z) \in[0, e] \times[0, e] \times \mathbb{L}$ then $U$ satisfies $(\mathbf{Q P})$.

(iii) $U$ satisfies $(\mathbf{Q P})$ on $[e, 1]^{2}$.

(iv) If $U(x, y) \in\{x \wedge y, x \vee y\}$ for all $(x, y) \in A(e)$ then $U$ satisfies $(\mathbf{Q P})$ on $A(e)$.

(v) Let $X \subseteq \mathbb{L}$ be such that $U(x, y) \in\{x, y\}$ for all $(x, y) \in X \times I_{e} \cup I_{e} \times X$. Then $U$ satisfies $(\mathbf{Q P})$ on $X \times I_{e} \cup I_{e} \times X$.

(vi) Let $U$ be a conjunctive uninorm and let $X, Y \subseteq \mathbb{L}$ such that $U(x, y)=0$ for all $(x, y) \in X \times Y$. Then $U$ satisfies $(\mathbf{Q P})$ on $X \times Y$.

(vii) Instead, let $U$ be a disjunctive uninorm and let $X, Y \subseteq \mathbb{L}$ such that $U(x, y)=1$ for all $(x, y) \in X \times$ $Y$. Then $U$ satisfies $(\mathbf{Q P})$ on $X \times Y$.

\subsection{Uninorms and (QP): A Pictorial Representation}

In Fig. 1, and the subsequent illustrations, we have employed the above partition.

The results given in Theorem 2 investigating the satisfaction of $(\mathbf{Q P})$ when the uninorm assumes different values in different regions are pictorially depicted in Fig. 1.

\section{Uninorms and (QP): For Specific Constructions}

In this section, we discuss the satisfaction of (QP) by the various constructions of uninorms on bounded lat-

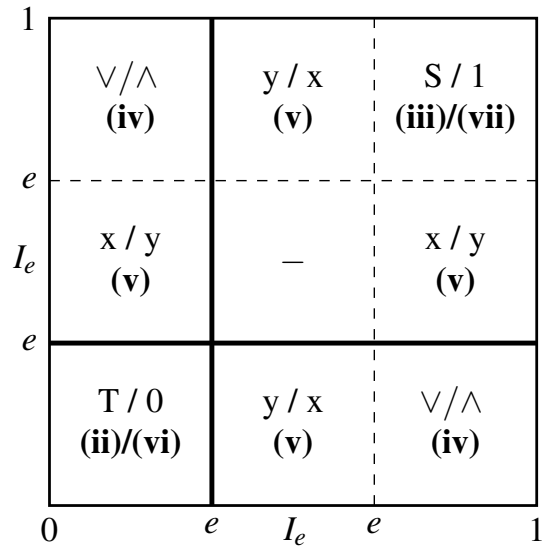

Figure 1: The representation of uninorms satisfying QP. The numbers in bold indicate the points in Theorem 2 in which the satisfaction of $(\mathbf{Q P})$ over that particular subdomain is discussed.

tices proposed in the literature. Our approach will be as follows:

- Typically every work proposes the construction of both a conjunctive and a disjunctive type of uninorms on bounded lattices. More often than not there is a duality present in the obtained construction. Hence, we discuss only one type of uninorms. WLOG, we discuss only the disjunctive type here.

- Further, bearing in mind the limitations in space, instead of presenting the detailed proof of the results we present the definitions of uninorms pictorially in terms of the operations defined on the different subdomains of $\mathbb{L} \times \mathbb{L}$. We believe this will allow the readers to quickly compare the diagramatic representation given in Fig. 1, thus enhancing both the readability and comprehension of the application of the results contained in Theorem 2 .

- However, we present examples of disjunctive uninorms from each of these constructions, along with the Hasse diagrams of the posets obtained from both the $\preceq_{U}$ and $\complement_{U}$ order relations.

- While there are many constructions of uninorms on bounded lattices, we have only chosen a few (five, to be precise). While this number has been fixed based on the available space, the choice itself has been dictated by either the generality or uniqueness of the construction proposed or for the interesting facets these reveal under the $\sqsubseteq$ order relation. 


\subsection{Different Constructions of Uninorms on a BL}

One of the earliest constructions of a uninorm on a bounded lattice is the work of Karaçal and Mesiar [11]. The uniqueness of this constrution is that one can make any element of $\mathbb{L}$ the identity of the constructed uninorm $U$.

Theorem 3 ([11], Theorem 1.). Let $(\mathbb{L}, \leq, 0,1)$ be a bounded lattice and $e \in \mathbb{L} \backslash\{0,1\}$. If $T_{e}$ is a $t$-norm on $[0, e]$ then the functions $U_{t}: \mathbb{L}^{2} \rightarrow \mathbb{L}$ defined as follows is a disjunctive uninorm on $\mathbb{L}$.

$U_{t}(x, y)= \begin{cases}T_{e}(x, y), & \text { if }(x, y) \in[0, e]^{2}, \\ x \vee y, & \text { if }(x, y) \in[0, e] \times(e, 1] \cup(e, 1] \times[0, e] \\ y, & \text { if } x \in[0, e] \text { and } y \| e, \\ x, & \text { ify } \in[0, e] \text { and } x \| e, \\ 1, & \text { otherwise }\end{cases}$

A pictorial representation of this disjunctive uninorm is given in Fig. 2.

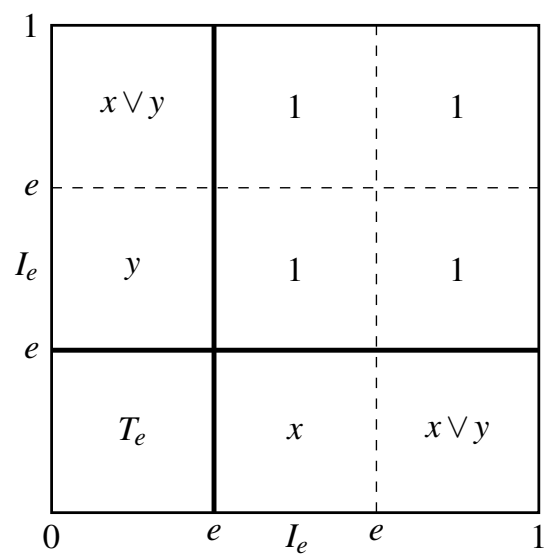

Figure 2: Pictorial representation of the disjunctive uninorm constructed in Theorem 3 ( [11]).

Proposition 3. Let $(\mathbb{L}, \leq, 0,1)$ be a bounded lattice, $e \in \mathbb{L} \backslash\{0,1\}$ and let $U_{t}$ be the uninorm as defined in Theorem 3. Then $\left(\mathbb{L}, \sqsubseteq U_{t}, 1, e\right)$ is a bounded $U_{t}$-poset.

Proof. From the pictorial representation of the uninorm $U_{t}$ in Fig. 2, comparing it with that of Fig. 1, we see that, since $U=T_{e}$, a t-norm on the region $[0, e] \times[0, e]$, the satisfaction of $(\mathbf{Q P})$ over this domain is guaranteed by Proposition 2 (ii).

Similarly, based on the definitions of $U_{t}$ on different regions, we immediately see that the satisfaction of $(\mathbf{Q P})$ on $[0, e] \times I_{e} \cup I_{e} \times[0, e]$ is shown in Theorem 2(v), while the same on $A(e)$ follows from Theorem 2(iv).

In the remaining regions, $U_{t} \equiv 1$ and the satisfaction of (QP) on this region follows from Proposition 2(vii).
Example 1. Consider a bounded lattice $L_{1}=$ $\{0, x, y, e, z, 1\}$ whose Hasse diagram is given in Figure 3. By using the construction method in Theorem 3, taking $T_{e}(x, y)=x \wedge y$ the uninorm $U_{t}: L^{2}{ }_{1} \rightarrow L_{1}$ is as defined in Table 1:

\begin{tabular}{c|c|c|c|c|c|c}
$U_{t}$ & 0 & $x$ & $y$ & $z$ & 1 & $e$ \\
\hline 0 & 0 & 0 & 0 & $z$ & 1 & 0 \\
\hline$x$ & 0 & $x$ & $x$ & $z$ & 1 & $x$ \\
\hline$y$ & 0 & $x$ & $y$ & $z$ & 1 & $y$ \\
\hline$z$ & $z$ & $z$ & $z$ & 1 & 1 & $z$ \\
\hline 1 & 1 & 1 & 1 & 1 & 1 & 1 \\
\hline$e$ & 0 & $x$ & $y$ & $z$ & 1 & $e$ \\
\hline
\end{tabular}

Table 1: The operation $U_{t}$ is a uninorm on $\left(L_{1}, \leq, 0,1\right)$ and a t-norm on $\left(L_{1}, \Xi_{t}, 1, e\right)$.

Note that while the original lattice $\left(L_{1}, \leq\right)$ is only bounded it is neither modular (consider the sublattice $\{0, e, y, z, 1\})$ nor a chain, whereas the obtained $U$-poset $\left(L_{1}, \sqsubseteq U_{t}\right)$ is, in fact, a chain and, hence, is distributive.
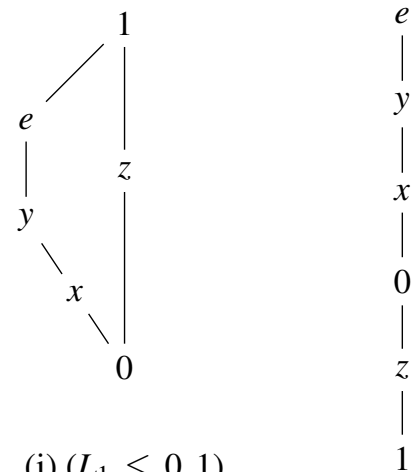

(i) $\left(L_{1}, \leq, 0,1\right)$

1

and $\left(L_{1}, \preceq_{U_{t}}, 0,1\right) \quad$ (ii) $\left(L_{1}, \sqsubseteq_{U_{t}}, 1, e\right)$

Figure 3: The lattice $\left(L_{1}, \leq, 0,1\right)$ and the $U$-poset obtained from the operation given in Table 1.

The next three constructions of uninorms are so chosen that readers can immediately verify the correctness of the claims using Theorem 2 and its pictorial representation.

Theorem 4 ([3], Theorem 1.). Let $(\mathbb{L}, \leq, 0,1)$ be a bounded lattice and $e \in \mathbb{L} \backslash\{0,1\}$. If $T_{e}$ is a $t$-norm on $[0, e]$ then the function $U^{t}: \mathbb{L}^{2} \rightarrow \mathbb{L}$ defined as follows is a disjunctive uninorm on $\mathbb{L}$ :

$$
U^{t}(x, y)= \begin{cases}T_{e}(x, y), & \text { if }(x, y) \in[0, e]^{2}, \\ y, & \text { if } x \in[0, e] \text { and } y \| e, \\ x, & \text { if } y[0, e] \text { and } x \| e, \\ x \vee y, & \text { otherwise }\end{cases}
$$




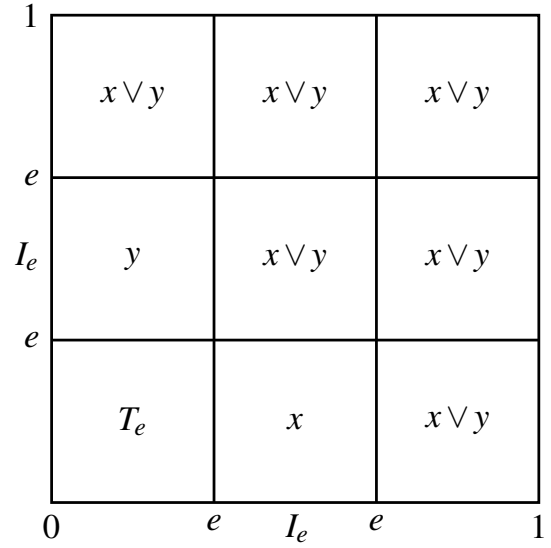

Figure 4: Pictorial representation of the disjunctive uninorm constructed in Theorem 4 ([3]).

A pictorial representation of this disjunctive uninorm is given in Fig. 4.

Proposition 4. Let $(\mathbb{L}, \leq, 0,1)$ be a bounded lattice, $e \in \mathbb{L} \backslash\{0,1\}$ and let $U^{t}$ be the uninorm as defined in Theorem 4. Then $\left(\mathbb{L}, \sqsubseteq_{U^{t}}, 1, e\right)$ is a bounded $U^{t}$-poset.

Example 2. Consider a bounded lattice $L_{2}=$ $\{0, e, x, y, z, t, 1\}$ whose Hasse diagram is given in Figure 5. By using the construction method in Theorem 4, taking $T_{e}(x, y)=x \wedge y$ the uninorm $U^{t}: L^{2}{ }_{2} \rightarrow L_{2}$ is as defined in Table 2:

\begin{tabular}{c|c|c|c|c|c|c|c}
$U^{t}$ & 0 & $x$ & $y$ & $z$ & $t$ & 1 & $e$ \\
\hline 0 & 0 & $x$ & $y$ & $z$ & $t$ & 1 & 0 \\
\hline$x$ & $x$ & $x$ & $z$ & $z$ & $t$ & 1 & $x$ \\
\hline$y$ & $y$ & $z$ & $y$ & $z$ & $t$ & 1 & $y$ \\
\hline$z$ & $z$ & $z$ & $z$ & $z$ & $t$ & 1 & $z$ \\
\hline$t$ & $t$ & $t$ & $t$ & $t$ & 1 & 1 & $t$ \\
\hline 1 & 1 & 1 & 1 & 1 & 1 & 1 & 1 \\
\hline$e$ & 0 & $x$ & $y$ & $z$ & $t$ & 1 & $e$ \\
\hline
\end{tabular}

Table 2: The operation $U^{t}$ is a uninorm on $\left(L_{2}, \leq, 0,1\right)$ and a t-norm on $\left(L_{2}, \sqsubseteq_{U^{t}}, 1, e\right)$.

Note that while the original lattice $\left(L_{2}, \leq\right)$ in Fig. 5 (i) is non-modular, the obtained $U^{t}$-poset - Fig. 5 (ii) - is, in fact, a distributive lattice $\left(L_{2}, \sqsubseteq_{U^{t}}\right)$.

Theorem 5 ([13], Theorem 5). Let $(\mathbb{L}, \leq, 0,1)$ be a bounded lattice, $e \in \mathbb{L} \backslash\{0,1\}$ and $x \| y$ for all $x \in I_{e}$ and $y \in[e, 1)$.. If $T_{e}$ and $S_{e}$ are $t$-norm and $t$-conorm on $[0, e]$ and $[e, 1]$, respectively. Then the function $U_{1, e}: \mathbb{L}^{2} \rightarrow \mathbb{L}$ defined as follows is a disjunctive uni-

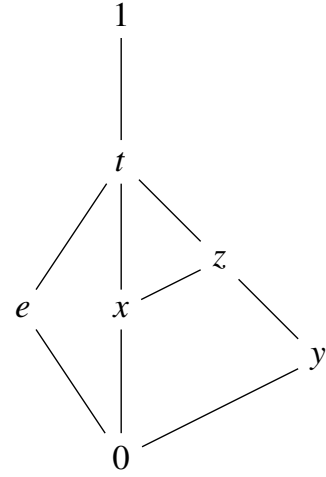

(i) $\left(L_{2}, \leq, 0,1\right)$ and
$\left(L_{2}, \preceq_{U^{t}}, 0,1\right)$
(ii) $\left(L_{2}, \sqsubseteq_{U^{t}}, 1, e\right)$

Figure 5: The lattice $\left(L_{2}, \leq, 0,1\right)$ and the $U$-poset obtained from the operation given in Table 2

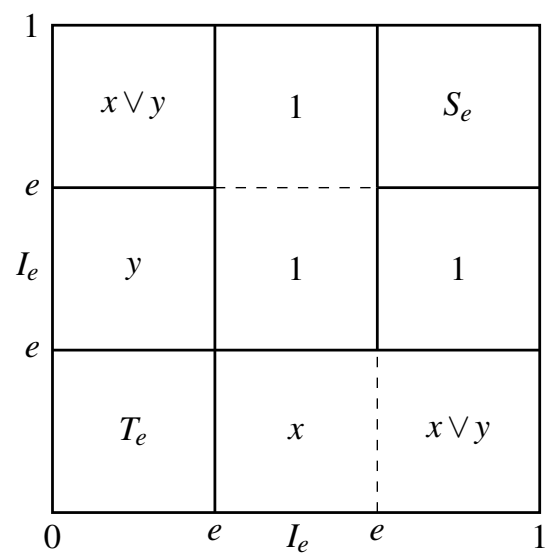

Figure 6: Pictorial representation of the disjunctive uninorm constructed in Theorem 5 ([13]).

norm on $\mathbb{L}$ :

$U_{1, e}(x, y)= \begin{cases}T_{e}(x, y), & \text { if }(x, y) \in[0, e]^{2}, \\ S_{e}(x, y), & \text { if }(x, y) \in[e, 1]^{2}, \\ y, & \text { if }(x, y) \in[0, e] \times I_{e}, \\ x, & \text { if }(x, y) \in I_{e} \times[0, e], \\ 1, & \text { if }(x, y) \in I_{e}{ }^{2} \cup I_{e} \times(e, 1] \cup(e, 1] \times I_{e}, \\ x \vee y, & \text { otherwise }\end{cases}$

A pictorial representation of this disjunctive uninorm is given in Fig. 6.

Proposition 5. Let $(\mathbb{L}, \leq, 0,1)$ be a bounded lattice, $e \in \mathbb{L} \backslash\{0,1\}$ and let $U_{1, e}$ be the uninorm as defined in Theorem 5. Then $\left(\mathbb{L}, \sqsubseteq_{1, e}, 1, e\right)$ is a bounded $U_{1, e^{-}}$ poset.

Example 3. Consider a bounded lattice $L_{3}=$ 


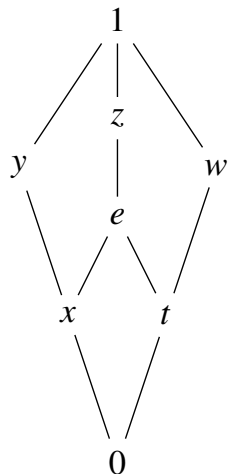

$\left(L_{3}, \leq, 0,1\right)$ and

$\left(L_{3}, \preceq_{U_{1, e}}, 0,1\right)$

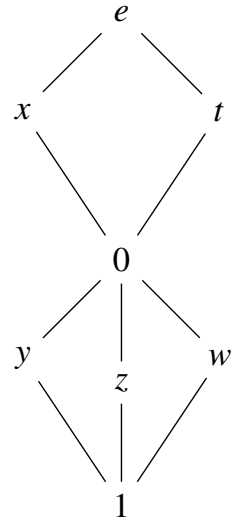

$\left(L_{3}, \sqsubseteq_{1, e}, 1, e\right)$
Figure 7: The lattice $\left(L_{3}, \leq, 0,1\right)$ and $U_{1, e}$-poset obtained from the operation given in Table 3

$\{0, x, y, z, t, w, e, 1\}$ whose Hasse diagram is given in Figure 7. By using the construction method in Theorem 5 , taking $T_{e}(x, y)=x \wedge y$ the uninorm $U_{1, e}: L_{3}^{2} \rightarrow$ $L_{3}$ is as defined in Table 3:

\begin{tabular}{c|c|c|c|c|c|c|c|c}
$U_{1 . e}$ & 0 & $x$ & $y$ & $z$ & $t$ & $w$ & 1 & $e$ \\
\hline 0 & 0 & 0 & $y$ & $z$ & 0 & $w$ & 1 & 0 \\
\hline$x$ & 0 & $x$ & $y$ & $z$ & 0 & $w$ & 1 & $x$ \\
\hline$y$ & $y$ & $y$ & 1 & 1 & $y$ & 1 & 1 & $y$ \\
\hline$z$ & $z$ & $z$ & 1 & 1 & $z$ & 1 & 1 & $z$ \\
\hline$t$ & 0 & 0 & $y$ & $z$ & $t$ & $w$ & 1 & $t$ \\
\hline$w$ & $w$ & $w$ & 1 & 1 & $w$ & 1 & 1 & $w$ \\
\hline 1 & 1 & 1 & 1 & 1 & 1 & 1 & 1 & 1 \\
\hline$e$ & 0 & $x$ & $y$ & $z$ & $t$ & $w$ & 1 & $e$ \\
\hline
\end{tabular}

Table 3: The Function $U_{1, e}$ is a uninorm on $\left(L_{3}, \leq\right)$ and a t-norm on $\left(L_{3}, \sqsubseteq U_{1, e}\right)$.

Theorem 6 ([2], Theorem 7). Let $(\mathbb{L}, \leq, 0,1)$ be a bounded lattice, $e \in \mathbb{L} \backslash\{0,1\}$ and $x \| y$ for all $x \in I_{e}$ and $y \in[e, 1)$. If $S_{e}$ is a t-conorm on $[0, e]$, then the function $U_{S_{e}}: \mathbb{L}^{2} \rightarrow \mathbb{L}$ defined as follows is a disjunctive uninorm on $\mathbb{L}$ :

$U_{S_{e}}(x, y)= \begin{cases}x \vee y, & \text { if }(x, y) \in A(e) \cup I_{e}{ }^{2}, \\ S_{e}(x, y), & \text { if }(x, y) \in[e, 1]^{2}, \\ y, & \text { if }(x, y) \in[0, e] \times I_{e}, \\ x, & \text { if }(x, y) \in I_{e} \times[0, e], \\ 1, & \text { if }(x, y) \in I_{e} \times(e, 1] \cup(e, 1] \times I_{e} \\ x \wedge y, & \text { otherwise }\end{cases}$

A pictorial representation of this disjunctive uninorm is given in Fig. 8.

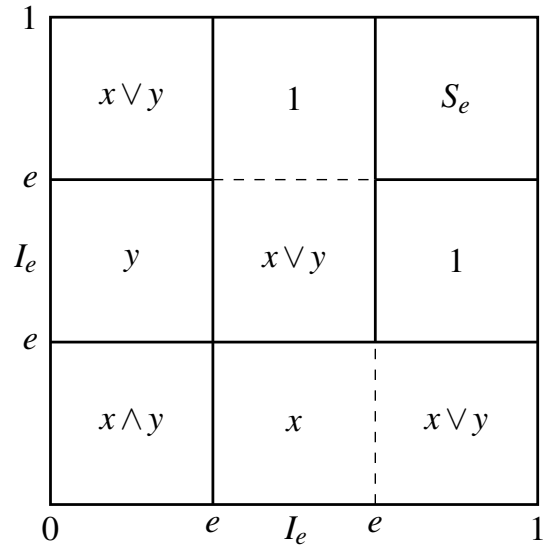

Figure 8: Pictorial representation of the disjunctive uninorm constructed in Theorem 6 ([2]).

Proposition 6. Let $(\mathbb{L}, \leq, 0,1)$ be a bounded lattice, $e \in \mathbb{L} \backslash\{0,1\}$ and let $U_{S_{e}}$ be the uninorm as defined in Theorem 6. Then $\left(\mathbb{L}, \sqsubseteq U_{S_{e}}, 1, e\right)$ is a bounded $U_{S_{e}}{ }^{-}$ poset.

Example 4. Consider a bounded lattice $L_{4}=$ $\{0, x, y, z, t, w, e, 1\}$ whose Hasse diagram is given in Figure 9. By using the construction method in Theorem 6, taking $S_{e}(x, y)=1$ on ]e,1] the uninorm $U_{S_{e}}$ : $L_{4}{ }^{2} \rightarrow L_{4}$ is as defined in Table 4 :

\begin{tabular}{c|c|c|c|c|c|c|c|c}
$U_{S_{e}}$ & 0 & $x$ & $y$ & $z$ & $t$ & $w$ & 1 & $e$ \\
\hline 0 & 0 & $x$ & $y$ & $z$ & $t$ & $w$ & 1 & 0 \\
\hline$x$ & $x$ & $x$ & $y$ & $z$ & 1 & 1 & 1 & $x$ \\
\hline$y$ & $y$ & $y$ & $y$ & $z$ & 1 & 1 & 1 & $y$ \\
\hline$z$ & $z$ & $z$ & $z$ & $z$ & 1 & 1 & 1 & $z$ \\
\hline$t$ & $t$ & 1 & 1 & 1 & 1 & 1 & 1 & $t$ \\
\hline$w$ & $w$ & 1 & 1 & 1 & 1 & 1 & 1 & $w$ \\
\hline 1 & 1 & 1 & 1 & 1 & 1 & 1 & 1 & 1 \\
\hline$e$ & 0 & $x$ & $y$ & $z$ & $t$ & $w$ & 1 & $e$ \\
\hline
\end{tabular}

Table 4: The Function $U_{S_{e}}$ is a uninorm on $\left(L_{4}, \leq\right)$ and a t-norm on $\left(L_{4}, \sqsubseteq U_{S_{e}}\right)$.

In this example, we can see that underlying lattice $\left(L_{4}, \leq, 0,1\right)$ and the obtained poset,$\preceq U_{S_{e}}$ and $\sqsubseteq U_{S_{e}}$ from the operation given in Table 4 are totally different.

Çayli et al. [4] proposed yet another construction, which is of interest to us due to the special type of lattices considered by them, see Theorem 7 . It can be shown that from the $U$ defined on such a lattice, we can always obtain a totally ordered poset using the $\sqsubseteq$ ordering relation.

Theorem 7 ([4], Theorem 3.18). Let $(\mathbb{L}, \leq, 0,1)$ be a bounded lattice, $e \in \mathbb{L} \backslash\{0,1\}$. If the subintervals $[0, e]$ 


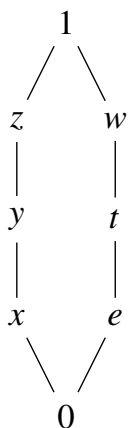

(i)

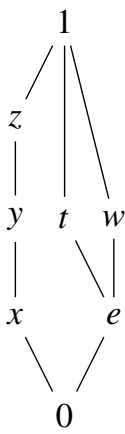

(ii)

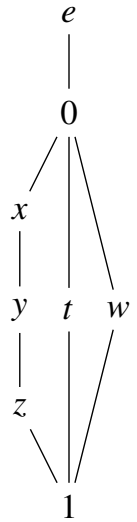

(iii)

$$
\left(L_{4}, \leq, 0,1\right) \quad\left(L_{4}, \preceq_{U_{S_{e}}}, 1, e\right) \quad\left(L_{4}, \sqsubseteq U_{S_{e}}, 1, e\right)
$$

Figure 9: The lattice $\left(L_{4}, \leq, 0,1\right)$ and $U_{S_{e}}$-poset obtained from the operation given in Table 4

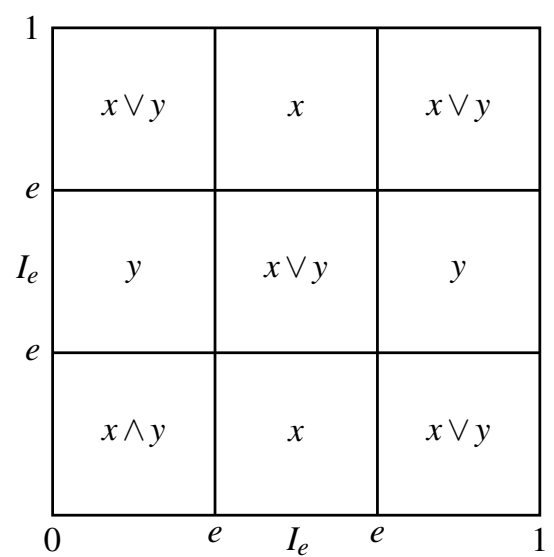

Figure 10: Pictorial representation of the disjunctive uninorm constructed in Theorem 7 ([4])

and $[e, 1]$ are chains and $x \| y$ for all $x \in I_{e}$ and $y \in$ $\mathbb{L} \backslash\{0,1\}$, then the function $U_{S, T}: \mathbb{L}^{2} \rightarrow \mathbb{L}$ defined as follows is a disjunctive uninorm on $\mathbb{L}$ :

$U^{S, T}(x, y)= \begin{cases}x \wedge y, & \text { if }(x, y) \in[0, e]^{2} \cup\{0\} \times[e, 1[ \\ y, & \text { or }\left[e, 1\left[\times\{0\} \cup\{0\} \times I_{e} \cup I_{e} \times\{0\},\right.\right. \\ x, & \text { if }(x, y) \in] 0, e] \times I_{e} \cup\left[e, 1\left[\times I_{e},\right.\right. \\ x \vee y, & \text { otherwise }\end{cases}$

A pictorial representation of this disjunctive uninorm is given in Fig. 10.

Proposition 7. Let $(\mathbb{L}, \leq, 0,1)$ be a bounded lattice, $e \in \mathbb{L} \backslash\{0,1\}$ and let $U^{S, T}$ be the uninorm as defined in Theorem 7. Then $\left(\mathbb{L}, \sqsubseteq_{U^{S, T}}, 1, e\right)$ is a total ordered bounded $U^{S, T}$-poset.

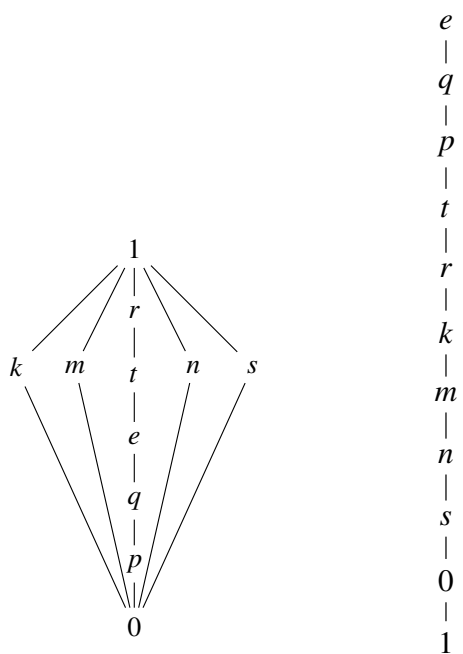

$$
\begin{aligned}
& \left(L_{5}, \leq, 0,1\right) \text { and } \\
& \left(L_{5}, \preceq_{U^{S, T}}, 0,1\right)
\end{aligned} \quad\left(L_{5}, \sqsubseteq_{U^{S, T}}, 1, e\right)
$$

Figure 11: The lattice $\left(L_{5}, \leq, 0,1\right)$ and $U^{S, T}$-poset obtained from the operation given in Table 5

Example 5. Consider a bounded lattice $L_{5}=$ $\{0, p, q, e, r, t, k, m, n, s, 1\}$ whose Hasse diagram is given in Figure 11. Note that the lattice $\left(L_{6}, \leq, 0,1\right)$ satisfies all constraints of Theorem 7. The uninorm $U^{S, T}: L^{2}{ }_{5} \rightarrow L_{5}$ is as defined in Table 5:

\begin{tabular}{c|c|c|c|c|c|c|c|c|c|c|c}
$U^{S, T}$ & 0 & $p$ & $q$ & $e$ & $k$ & $m$ & $n$ & $s$ & $t$ & $r$ & 1 \\
\hline 0 & 0 & 0 & 0 & 0 & 0 & 0 & 0 & 0 & 0 & 0 & 1 \\
\hline$p$ & 0 & $p$ & $p$ & $p$ & $k$ & $m$ & $n$ & $s$ & $t$ & $r$ & 1 \\
\hline$q$ & 0 & $p$ & $q$ & $q$ & $k$ & $m$ & $n$ & $s$ & $t$ & $r$ & 1 \\
\hline$e$ & 0 & $p$ & $q$ & $e$ & $k$ & $m$ & $n$ & $s$ & $t$ & $r$ & 1 \\
\hline$k$ & 0 & $k$ & $k$ & $k$ & $k$ & $m$ & $n$ & $s$ & $k$ & $k$ & 1 \\
\hline$m$ & 0 & $m$ & $m$ & $m$ & $m$ & $m$ & $n$ & $s$ & $m$ & $m$ & 1 \\
\hline$n$ & 0 & $n$ & $n$ & $n$ & $n$ & $n$ & $n$ & $s$ & $n$ & $n$ & 1 \\
\hline$s$ & 0 & $s$ & $s$ & $s$ & $s$ & $s$ & $s$ & $s$ & $s$ & $s$ & 1 \\
\hline$t$ & 0 & $t$ & $t$ & $t$ & $k$ & $m$ & $n$ & $s$ & $t$ & $r$ & 1 \\
\hline$r$ & 0 & $r$ & $r$ & $r$ & $k$ & $m$ & $n$ & $s$ & $r$ & $r$ & 1 \\
\hline 1 & 1 & 1 & 1 & 1 & 1 & 1 & 1 & 1 & 1 & 1 & 1 \\
\hline
\end{tabular}

Table 5: The Function $U^{S, T}$ is uninorm on $\left(L_{5}, \leq\right)$ and t-norm on $\left(L_{5}, \sqsubseteq_{U S, T}\right)$.

Note that while the original lattice $\left(L_{5}, \leq\right)$ is only modular not a chain, the obtained $U^{S, T}$-poset $\left(L_{5}, \sqsubseteq_{U^{S, T}}\right)$ is, in fact, a chain and, hence, is distributive.

\section{The $\sqsubseteq$-relation on Uninorms: Some Interesting Facets}

In this concluding section, we present some interesting perspectives that arise out of our study, thus both vindicating and highlighting the investigations contained in this submission. 


\subsection{Uninorms as T-norms on the Obtained Posets}

It is well known that a uninorm is a generalisation of a t-norm and a t-conorm. Algebraically speaking, both a uninorm and a t-norm on a bounded lattice $(\mathbb{L}, \leq, 0,1)$ are commutative ordered monoids. However, additionally a t-norm is also an integral monoid, i.e., the identity element is also the top element of the bounded lattice, whereas by construction the identity $e \in \mathbb{L} \backslash\{0,1\}$.

Interestingly, as shown above, the poset obtained from $\sqsubseteq U$ is again a bounded poset with $e$ as the top element and the given uninorm $U$ remains order preserving on the poset $\left(\mathbb{L}, \sqsubseteq_{U}, 0, e\right)$ due to its commutativity. Thus, on this obtained bounded poset $\left(\mathbb{L}, \sqsubseteq_{U}, 0, e\right)$ a uninorm is also an integral commutative ordered monoid, which implies the $U$ can be considered as a t-norm on $(\mathbb{L}, \sqsubseteq U$ $, 0, e)$.

Recently, there have been a spate of works on constructing t-norms on bounded posets. While currently the authors do not have a mechanism to engineer ondemand structures on the obtained poset, we hope such perspectives will be useful to these studies.

\subsection{Possibility for Richer Obtained Posets}

As the discussion above has shown, often, the obtained poset $\left(\mathbb{L}, \sqsubseteq_{U}, 0, e\right)$ seems to satisfy further desirable properties, like modularity, distributivity or linearity, than the original underlying poset $(\mathbb{L}, \leq, 0,1)$ considered.

In the literature, one finds different ways of linearising a given poset - for instance, using a topological sort, in which case it becomes both distributive, and hence modular too. While the current procedure can hardly be compared with general purpose procedures as cited above, our study is not bereft of value.

For instance, from the construction of Karaçal and Mesiar [11], one can choose as $e$ an element that is maximally non-related to any other element in $(\mathbb{L}, \leq)$, i.e., $e \in \mathbb{L}$ and $e \| x$ for all $x \in \mathbb{L} \backslash\{0,1\}$. Now, using the $\sqsubseteq$ order, this element will be the top element and hence will be maximally related to every other element.

Further, as Proposition 7 shows, one can even present some general results stating the conditions underwhich we can expect richer structures on $\left(\mathbb{L}, \sqsubseteq_{U}, 0, e\right)$. In fact, it can be shown that every internal uninorm on a bounded lattice makes $(\mathbb{L}, \sqsubseteq U, 0, e)$ a total order - of course, the converse is not true. Clearly, further investigations along this line are worthy of study and are already underway.

\section{Acknowledgement}

The work described in this article was supported by
University Grants Commission (UGC), New Delhi, India (Ref. No. 20/12/2015(ii)EU-V). The second author would like to gratefully acknowledge the partial support of SERB under the project MTR/2020/000506.

\section{References}

[1] E. Aş1c1, An order induced by nullnorms and its properties, Fuzzy Sets and Systems 325 (2017) $35-46$.

[2] E. Aşıcı, R. Mesiar, On the construction of uninorms on bounded lattices, Fuzzy Sets and Systems.

[3] G. D. Çaylı, F. Karaçal, R. Mesiar, On a new class of uninorms on bounded lattices, Information Sciences 367 (2016) 221-231.

[4] G. D. Çaylı, F. Karaçal, R. Mesiar, On internal and locally internal uninorms on bounded lattices, International Journal of General Systems 48 (3) (2019) 235-259.

[5] A. H. Clifford, Naturally totally ordered commutative semigroups, American Journal of Mathematics 76 (3) (1954) 631-646.

[6] Ü. Ertuğrul, M. N. Kesicioğlu, F. Karacal, Ordering based on uninorms, Information Sciences 330 (2016) 315-327.

[7] V. K. Gupta, B. Jayaram, Importation algebras, in: International Summer School on Aggregation Operators, Springer, 2019, pp. 83-94.

[8] V. K. Gupta, B. Jayaram, Importation lattices, Fuzzy Sets and Systems 405 (2021) 1-17.

[9] V. K. Gupta, B. Jayaram, Order based on associative operations, Information Sciences 566 (2021) 326-346.

[10] F. Karaçal, M. N. Kesicioğlu, A T-partial order obtained from t-norms, Kybernetika 47 (2) (2011) 300-314.

[11] F. Karaçal, R. Mesiar, Uninorms on bounded lattices, Fuzzy Sets and Systems 261 (2015) 33-43.

[12] M. N. Kesicioğlu, R. Mesiar, Ordering based on implications, Information Sciences 276 (2014) 377-386.

[13] A. Xie, S. Li, On constructing the largest and smallest uninorms on bounded lattices, Fuzzy Sets and Systems 386 (2020) 95-104. 